\title{
Contemporary Natural Philosophy and Philosophies
}

\author{
Gordana Dodig-Crnkovic ${ }^{1,2, *(1)}$ and Marcin J. Schroeder ${ }^{3}$ \\ 1 Department of Computer Science and Engineering, Chalmers University of Technology, \\ 41296 Gothenburg, Sweden \\ 2 School of Innovation, Design and Engineering, Computer Science Laboratory, Mälardalen University, \\ 72220 Västerås, Sweden \\ 3 Akita International University, 193-2 Okutsubakidai, Aza Tsubakigawa, Yuwa, 010-1211 Akita, Japan; \\ mjs@aiu.ac.jp \\ * Correspondence: dodig@chalmers.se
}

Received: 21 November 2018; Accepted: 22 November 2018; Published: 26 November 2018

\begin{abstract}
In this Editorial note, Guest Editors introduce the theme of the Special Issue of the journal Philosophies, titled Contemporary Natural Philosophy and Philosophies.
\end{abstract}

Keywords: natural philosophy; philosophy of nature; naturalism; unity of knowledge

\section{Introduction}

From the Philosophies program [1], one of the main aims of the journal is to help establish a new unity in diversity in human knowledge, which would include both "Wissen" (i.e., "Wissenschaft") and "scire" (i.e., "science"). As is known, "Wissenshaft" (the pursuit of knowledge, learning, and scholarship) is a broader concept of knowledge than "science", as it involves all kinds of knowledge, including philosophy, and not exclusively knowledge in the form of directly testable explanations and predictions. The broader notion of scholarship incorporates an understanding and articulation of the role of the learner and the process of the growth of knowledge and its development, rather than only the final product and its verification and validation. In other words, it is a form of knowledge that is inclusive of both short-term and long-term perspectives; it is local and global, critical and hypothetical (speculative), breaking new ground. This new synthesis or rather re-integration of knowledge is expected to resonate with basic human value systems, including cultural values.

Since knowledge tends to spontaneously fragment while it grows, Philosophies takes existing diversity as a resource and a starting point for a new synthesis. The idea of broad, inclusive knowledge is in fact not so new. From the beginning, natural philosophy included all contemporary knowledge about nature. Newton was a natural philosopher, as were Bohr, Einstein, Prigogine, Weizsäcker, and Wheeler-to name but a few. Today, the unifying picture of the natural/physical world is sorely missing among the isolated silos of particular scientific domains, each with its own specific ontologies, methodologies, and epistemologies.

From the profound need for connected and common knowledge, new trends towards synthesis have emerged in the last decades. One major theme is complexity science, especially when applied to biology or medicine, which helps us to grasp the importance of connectedness between present-day disparate pieces of knowledge-frameworks, theories, approaches, etc. Related to this is the emergence of network science, which studies structures of nodes (actors) and edges as connections between them.

In an adage ascribed to Einstein, but also some others such as Hawkins, it has been recognized that problems are solved not in the framework in which they appear but rather in a new framework, at the next level of abstraction.

This Special Issue responds to the call from Philosophies to build a new, networked world of knowledge with domain specialists from different disciplines interacting and connecting 
with the rest of knowledge-producing and knowledge-consuming communities in an inclusive, extended natural-philosophic manner. In this process of synthesis, scientific and philosophical investigations enrich each other-with sciences informing philosophies about the best current knowledge of the world, both natural and human-made-while philosophies scrutinize the ontological, epistemological, and methodological foundations of sciences, providing scientists with questions and conceptual analyses. This is all directed at extending and deepening our existing comprehension of the world, including ourselves, both as humans and as societies, and humankind.

\section{Obstacles to a New Synthesis}

Historically, attempts were made to search for a unity of knowledge originating from insights into the need to understand the world in a holistic manner, notably Snow's critique of "The Two Cultures" [2] and "Consilience: The Unity of Knowledge" by biologist Wilson [3]. However, the strong development of disciplinary research continued as if nothing had happened. It was still possible to continue to dig deeper into isolated domains, and the results were still interesting even though a common view was missing. However, new developments in sciences and technology, such as artificial intelligence, neurosciences, and cognitive science, called for unified views of the "body" and mind", the physical and the mental as archetypes of the divide between "two cultures".

The dialogue between sciences and philosophy has become especially interesting when it comes to the philosophy of science and the question of what constitutes the scientific method, which has become less and less clear. There are three major methodological challenges:

- The demise of natural philosophy: this is a very conservative position, still quite common, held by those who believe, as was fashionable in the late 19th and early 20th centuries, that science needs to emancipate itself from the "philosophical nonsense" that conflates philosophy with metaphysics, where metaphysics is understood as a priori knowledge about the nature of reality. Philosophy is of course much more than metaphysics understood in this narrow sense. Recently, a strong interest in ontology and epistemology within artificial intelligence and robotics has demonstrated how important those branches of metaphysics can be not only for science but even for technology. The study of space and time, causality, necessity, and chance are other examples where sciences (physics, biology) expand into traditional territories of metaphysics.

- "Idol of Numbers": today, this can be added to Bacon's four Idols of the Mind (Idols of the Tribe, Idols of the Cave, Idols of the Marketplace, and Idols of the Theater) [4]. This is not less conservative, and possibly even more dangerous in the era of "big data" and data-driven science. Followers of this cult dismiss everything that is not presented in terms of numbers and trust only in the "objective character" of that which is given in numerical form, for example, as expressed in the maxim "let the data speak for themselves". It became more important "that" we can provide numerical values than "what" these numerical values represent and "what" these numbers tell us about reality.

- Isolationism and the self-sufficiency of research disciplines: Along with the previous two obstacles to this new synthesis, a third, associated one must also be added. This relates to the difficulty of communication between different domains of knowledge, which makes the role of interdisciplinarity/crossdisciplinarity and transdisciplinarity central to the construction of our contemporary knowledge of the world.

\section{Possible Avenues of Re-Connection}

When modeling a phenomenon, multiple connected theories, seen from a common perspective, contribute to our multifaceted understanding of its structures and temporal behavior.

One very successful approach in this direction was the development of multiscale models for complex physical, chemical, biological, and cognitive systems, including the human brain. Multiscale 
models [5] combine and connect earlier approaches focused on single scales of time, space, and topology through the integration of data across spatial, temporal, and functional scales.

Another promising path is the reconceptualization (i.e., conceptual engineering) of the basic concepts used to describe different natural and artifactual systems-physical, chemical, biological, and cognitive. In this new framework, information is considered as the fabric of reality (Deutsch) [6], for an observer, Floridi [7]. The dynamics of information can be modeled as computation, thus forming the basis for the info-computational modeling of a variety of systems, from the physical to the cognitive [8]. According to Kun Wu and Brenner [9], the philosophy of information presents a revolution in philosophy and provides a means of informational metaphilosophy of science that is philosophy of the philosophy of science. We might also add that information, together with its dynamics (computation), presents a new possibility for the development of the modern philosophy of nature/natural philosophy.

\section{Possible Topics of Interest}

For this Special Issue, we called for contributions on a variety of topics, as set out in, but not limited to, the following list:

- What is the current state of the philosophy of nature / natural philosophy?

- What might be the role of the philosophy of nature / natural philosophy?

- Can the philosophy of nature be based on our best current scientific knowledge? (the thesis of the book "Everything Must Go" [10]);

- How can interdisciplinarity/crossdisciplinarity/ multidisciplinarity/transdisciplinarity help tie knowledge from different disciplines and interdisciplines at different levels of abstraction in a common intelligible philosophy of the universe with cosmos and chaos, non-living and living parts in it? [11,12]

- What would be the new role of research methods in this new high-level take on human knowledge?

- Can we imagine any higher authority in matters of truth and existence than the consensus view of our current humanity?

- How do the sciences of the artificial [13], AI, relate to the philosophy of nature?

- Informational universe-Floridi-Deutsch—Wu Kun-epistemology—ontology;

- "Mechanism" and "materialism" as bases for our understanding of nature;

- Nature and mind - the role and character of the mind/cognition agency in the development of the universe;

- Evolving universe-being and becoming in contemporary philosophy of nature;

- Emergent universe;

- Connecting a variety of levels of abstraction;

- The role of life sciences, with biology and cognitive sciences, in the new natural philosophy;

- The role of the observer in the new synthesis;

- The role of formal sciences and methods-logics, mathematics, computing, simulation;

- The ecological view of knowledge [14].

This list could be made much longer to address the variety of aspects of the new synthesis in contemporary natural philosophy, but its role was only to illustrate the goal of this Special Issue.

We consider the present issue as only the first step towards a more organized and sustainable collective effort to revive the original fundamental role of natural philosophy, construed as the pursuit of integrated knowledge and understanding of the world. We hope that this issue will generate sufficient interest and support to continue publishing similar special issues in the future. 
Acknowledgments: The Guest Editors would like to express their gratitude to the authors that contributed to this Special Issue. They were overwhelmed by the response, both in terms of the number of submissions as well as their wide range of topics and excellent quality. They hope that this Special Issue will contribute to the new synthesis in the form of a revived modern natural philosophy.

Conflicts of Interest: The authors declare no conflict of interest.

\section{References}

1. Schroeder, M.J. The Philosophy of Philosophies: Synthesis through Diversity. Philosophies 2016, 1, 68. [CrossRef]

2. Snow, P.C. The Two Cultures; Cambridge University Press: Cambridge, UK, 2001/1959; ISBN 0-521-45730-0.

3. Wilson, E.O. Consilience. The Unity of Knowledge; Knopf/ Random House: New York, NY, USA, 1998.

4. Bacon, F. The New Organon; Book 1. The Works of Francis Bacon; Spedding, J.; Ellis, R.L.; Heath, D.D., Translators; Taggard and Thompson: Boston, MA, USA, 1863; Volume 8.

5. Weinan, E. Principles of Multiscale Modeling; Cambridge University Press: Cambridge, UK, 2011.

6. Deutsch, D. The Fabric of Reality; Penguin Books: New York, NY, USA, 1997.

7. Floridi, L. A defence of informational structural realism. Synthese 2008, 161, 219-253. [CrossRef]

8. Dodig Crnkovic, G. Nature as a Network of Morphological Infocomputational Processes for Cognitive Agents. Eur. Phys. J. 2017, 226, 181-195. [CrossRef]

9. Wu, K.; Brenner, J. Philosophy of Information: Revolution in Philosophy. Towards an Informational Metaphilosophy of Science. Philosophies 2017, 2, 22. [CrossRef]

10. Ladyman, J.; Ross, D.; Spurrett, D.; Collier, J. Every Thing Must Go: Metaphysics Naturalized; Oxford UP: Oxford, UK, 2007; ISBN 978-0199573097.

11. Hofkirchner, W. (Ed.) The Quest for a Unified Theory of Information; Proceedings of the Second International Conference on the Foundations of Information Science. World Futures General Evolution Studies; Gordon and Breach Publ: Amsterdam, The Netherlands, 1999.

12. Brier, S. Cybersemiotics: Why Information Is Not Enough; University of Toronto Press: Toronto, ON, Canada, 2008.

13. Simon, H.A. The Sciences of the Artificial; MIT Press: London, UK, 1996.

14. Burgin, M.; Zhong, Y. Information Ecology in the Context of General Ecology. Information 2018, 9, 57. [CrossRef]

(C) 2018 by the authors. Licensee MDPI, Basel, Switzerland. This article is an open access article distributed under the terms and conditions of the Creative Commons Attribution (CC BY) license (http:/ / creativecommons.org/licenses/by/4.0/). 